\title{
SIMULATION AND EXAMINATION OF A SIGNAL MASKING CHAOTIC COMMUNICATION SYSTEM, BASED ON THE DUFFING OSCILLATOR
}

The study in this paper is focused on the applications of the chaotic Duffing oscillator to signal masking communication scheme. Using the concept of synchronized chaotic systems the signal masking approach is demonstrated with the Duffing oscillator implemented in both the transmitter and receiver. The chaotic masking signal is added at the transmitter to the information signal, and at the receiver, the masking is regenerated and subtracted from the received signal. Chaotic behavior and chaotic masking scheme are designed and simulated using Simulink/Matlab program. All the results are used to illustrate the effectiveness and the applicability of the Duffing oscillator in signal masking communication.

Keywords: Chaotic signal, chaotic communication system, chaotic masking, Duffing oscillator, error signal.

\section{Introduction}

During the last years the use of chaos in communications has become a very important topic of research. Chaos is applied in communications solutions especially in the context of channel encoding and modulation. Observing the ongoing research [1, 2], one observes that the vast majority of the schemes proposed so far concerns modulation in the first place, whereas only a few consider the channel encoding. Chaotic masking [3, 4, 5], switching [6, 7], parameter modulation $[8,9]$ are some of the methods, proposed in the literature to send binary messages.

Chaotic signal masking is an approach with application in the secure communication $[2,3,7]$. It is based on chaos synchronization $[1,3,5,10]$. The synchronized motion in coupled oscillator systems is first discussed by Fujisaka and Yamada [1]. The most effective and widely studied approach is due to Pecora and Carroll's discovery $[5,8]$. They have reported that certain chaotic systems possess a selfsynchronization property.

The Duffing non-autonomous oscillator $[6,11]$ is a classical example of a nonlinear dynamical system representing chaotic behavior. The Duffing oscillator has synchronization property and it can be used to the signal masking communication scheme.

In this paper synchronization between two chaotic systems, based on the Duffing oscillator is proposed. The chaotic systems are applied to masking approach in sending secret messages. The communication scheme is simulated using Simulink/Matlab program. The simulation results in this paper investigate the circum- stances of application of the Duffing oscillator to drive and response chaotic systems in signal masking communication scheme.

\section{Chaotic masking approach description}

The chaotic signal masking approach is based on the identical synchronization, where the state of response system converges asymptotically to the state of the drive system [6].

Two continuous-time chaotical systems

$$
x^{(1)}=f(x, t)
$$

and

$$
x^{(2)}=f(\tilde{x}, t)
$$

are said to synchronize identically if

$$
\lim _{t \rightarrow \infty}\left(x^{(2)}-x^{(1)}\right)=0
$$

for any combination of initial states $[4,7]$.

In the equations (1) and (2) $x, \tilde{x}$ are according the vectors of the state of the two systems, $t$ - the time.

The system (1) is located in the transmitter and system (2) in the receiver (Fig. 1). If the same initial condition is chosen for the transmitter and the receiver, the both systems will evolve in a syn-

\footnotetext{
* Galina Cherneva, Elena Dimkina

Faculty of Telecommunications and Electrical Equipment in Transport, Kableshkov University of Transport, Sofia, Bulgaria, E-mail: cherneva@vtu.bg
} 
chrony in the sense that, $x^{(1)}$ will continue being equal to $x^{(2)}$ for all $t>0$.

The idea of a chaotic masking approach is that the information signal is masked by directly adding a chaotic signal at the transmitter. Because chaotic signals are typically broadband, noise like, and difficult to predict, they can be used for masking informationbearing waveforms.

At the receiver which is synchronized to the transmitter the chaotic component is subtracted from the received signal to recover the original transmitted message.

The transmitter state evolution is given by the chaotic dynamics

$$
F(x(t))
$$

A chaotic signal $u(t)$ which is a function of the transmitter state $x(t)$ is added to the information signal $m(t)$.

The transmitted signal $s(t)$ is governed by

$$
s(t)=u(t)+m(t)
$$

The evolution of the receiver state $x_{r}(t)$ dynamics is given by the dynamics

$$
\frac{d x_{r}}{d t}=F\left(x_{r}(t), s(t)\right) \text {. }
$$

and $v(t)$ is the chaotic signal which is a function of the receiver state $x_{r}(t)$.

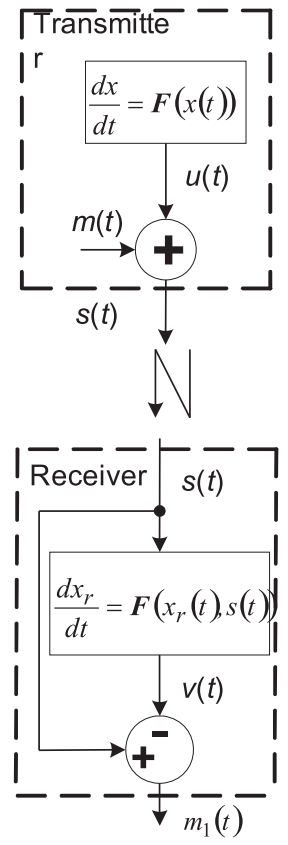

Fig. 1 Block diagram of a chaotic masking communication system
Then the combined signal (5), received by the receiver, can be used to regenerate the chaotic signal $u(t)$ through chaos synchronization. If the synchronization is successful, the $v(t)$ converge to $u(t)$ after a short initial transient and the original message can be recovered by subtracting the reproduced chaotic signal $v(t)$ from the received signal $s(t)$ :

$$
m_{1}(t)=s(t)-v(t) \approx s(t)-v(t)=m(t) .
$$

The masking scheme works only when the amplitudes of the information signals are much smaller than the masking chaotic signals

$$
m(t)<<|u(t)|
$$

The systems with chaotic masking works effectively in small disturbances in the channel (high ratio signal/noise).

\section{Model construction}

The Duffing oscillator is given by the second-order differential equation [4] with an external periodic drive term:

$$
\ddot{x}+a \dot{x}-x+x^{3}=A \sin \omega t,
$$

or by an equivalent set of two first order non -autonomous equations

$$
\mid \begin{aligned}
& \frac{d x}{d t}=y \\
& \frac{d y}{d t}=x-x^{3}-a y+A \sin \omega t
\end{aligned},
$$

where $a, A$ and $\omega$ are parameters.

The transmitter state variables are denoted by the vector

$$
d=(x, y)
$$

and the receiver variables by the vector

$$
r=\left(x_{r}, y_{r}\right)
$$

Assume that the transmitter is described by the equation (10). A response system that will synchronize to the chaotic signals at the transmitter (10) is described by

$$
\mid \begin{aligned}
& \frac{d x_{r}}{d t}=y_{r}-\delta\left(x-x_{r}\right) \\
& \frac{d y_{r}}{d t}=x_{r}-x_{r}^{3}=a y+A \sin \omega t
\end{aligned}
$$

where $\delta$ is a coupling parameter to couple the receiver with the transmitter.

This system is obtained from the transmitter equations by renaming variables $(x, y)$ to $\left(x_{r}, y_{r}\right)$. 
The synchronization between the transmitter (10) and receiver (13) has been proved by analyzing the stability of the error signal. It is derived from the difference between the output of the receiver system and that received from the transmitter:

$$
e=d-r=\left(e_{1}, e_{2}\right)=\left(x-x_{r}, y-y_{r}\right)
$$

The error signal is then used to modify the state of the receiver such that it can be synchronized with the transmitter. This signal is given by the system

$$
\text { with } \mid \begin{aligned}
& \dot{e}_{1}=e_{2}-\delta e_{1} \\
& \dot{e}_{2}=e_{1}-e_{1}\left(x^{2}+x x_{r}+x_{r}^{2}\right)-a e_{2}
\end{aligned} .
$$

The characteristic equation of system (15) is

$$
\lambda^{2}+a_{1} \lambda+a_{2}=0
$$

where

$$
\begin{aligned}
& a_{1}=\delta+a, \\
& a_{2}=\delta a+x^{2}+x x_{r}+x_{r}^{2}-1 . \\
& \text { If } a_{1}>0 \text { and } a_{2}>0, \text { then } \\
& e_{1}=e_{2}=0
\end{aligned}
$$

is a stable fixed point [3] and the receiver will eventually synchronize with the transmitter.

$$
\text { If } a>0 \text { and } \delta>0 \text {, }
$$

then

$$
a_{1}>0
$$

Since

$$
x^{2}+x x_{r}+x_{r}^{2}=\left(x+\frac{1}{2} x_{r}\right)^{2}+\frac{3}{4} x_{r}^{2}>0,
$$

so $a_{2}$ can also be positive if

$$
\delta \geq \frac{1}{a}
$$

\section{Simulation results}

Chaotic signal masking communication scheme, based on the Duffing oscillator, is designed and simulated using Simulink/Matlab program. Figure 2 shows the simulation scheme. The information signal $m(t)$ is a sinus wave of amplitude 1 volts and of $5 \mathrm{kHz}$ frequency.

Transmitter and receiver systems are identical. In the present case $a=0.2$ is accepted.

As follows of (19) the transmitter and receiver are synchronized when $\delta \geq 5$.

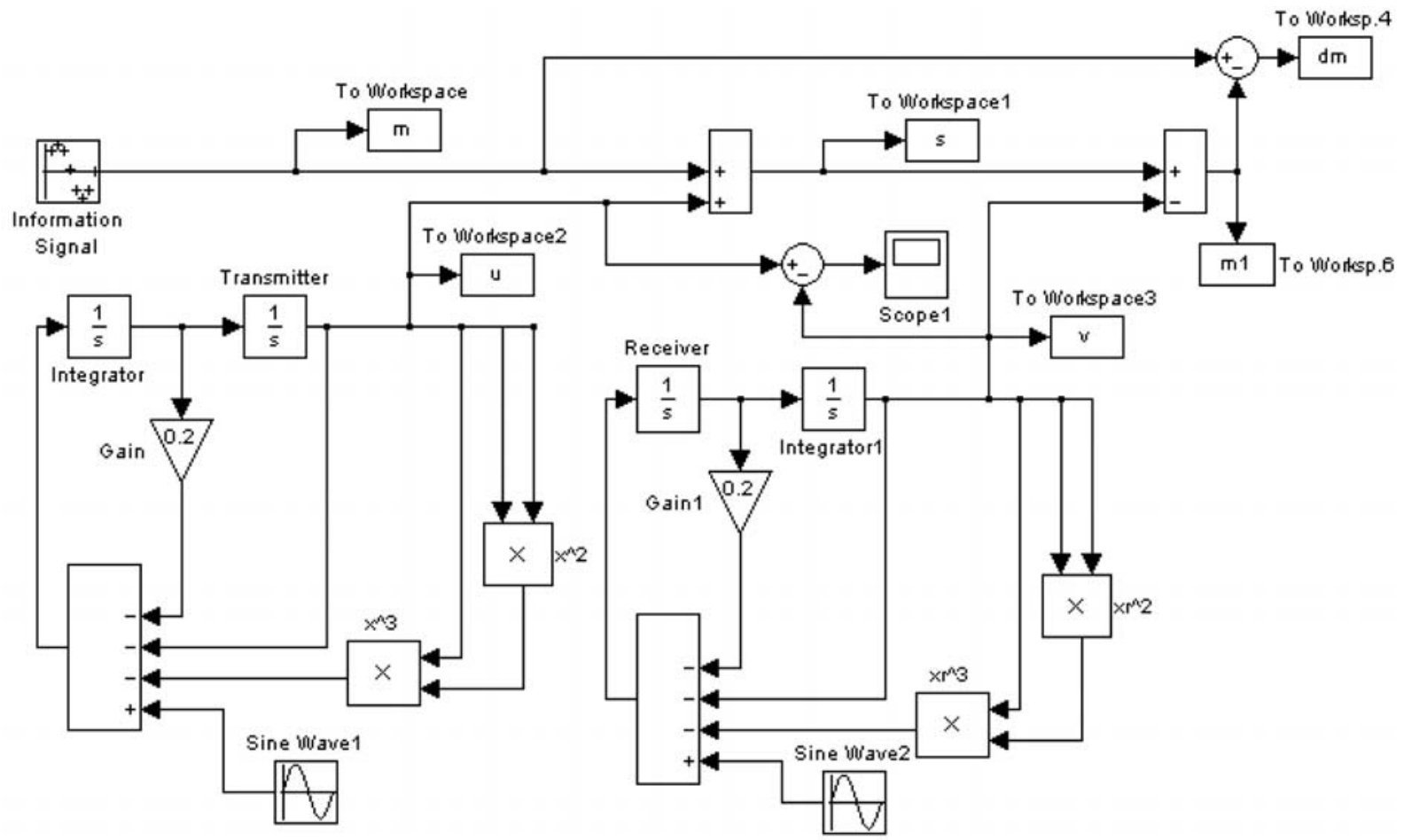

Fig. 2 Simulink modeling of chaotic masking scheme of the Duffing oscillator 
In the following analysis the synchronization between the transmitter and the receiver is obtained with an absence of disturbance.

The information signal is added to the generated chaotic signal $u(t)$, and the signal

$s(t)=m(t)+u(t)$

is fed into the receiver.

The chaotic signal $v(t)$ is generated in receiver system and

$m_{1}(t)=s(t)-v(t) \approx m(t)$

if

$$
u(t)=v(t)
$$

The examination is based on a simulation of the systems in the transmitter and the receiver unto different initial conditions and comparison of the driving signal $u(t)$ with the response signal $v(t)$.

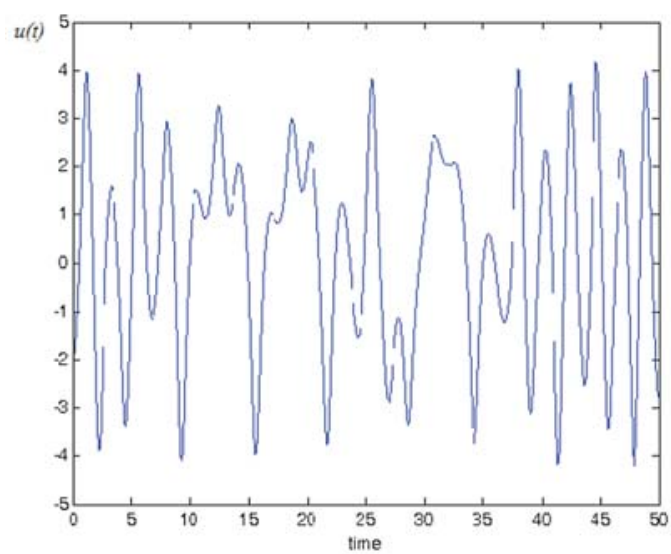

Fig. 3 Phase plane plot of the transmitter chaotic signal

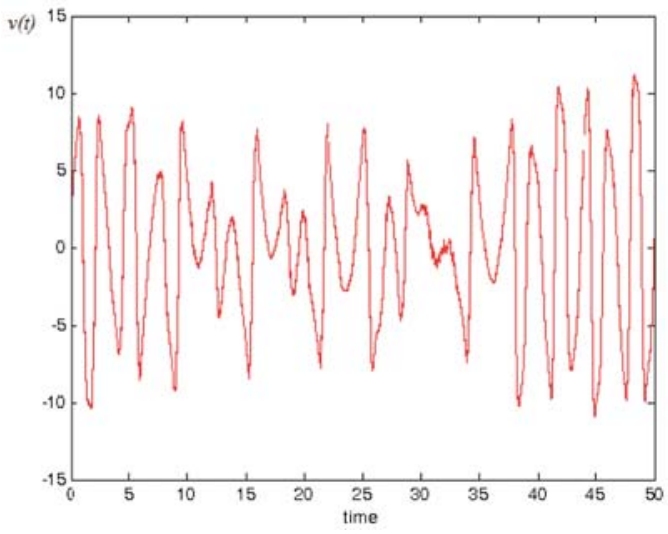

Fig. 4 Phase plane plot of the receiver chaotic signal
The phase plane of the transmitter and receiver chaotic signals is shown in Figs. 3 and 4.

Figure 5 shows the difference $u(t)-v(t)$ of scope.

It is seen that after a transient period the error signal of the transmitter and receiver systems goes to zero, showing that the two systems are synchronized.

One can see from the figures that the synchronization is satisfactory.

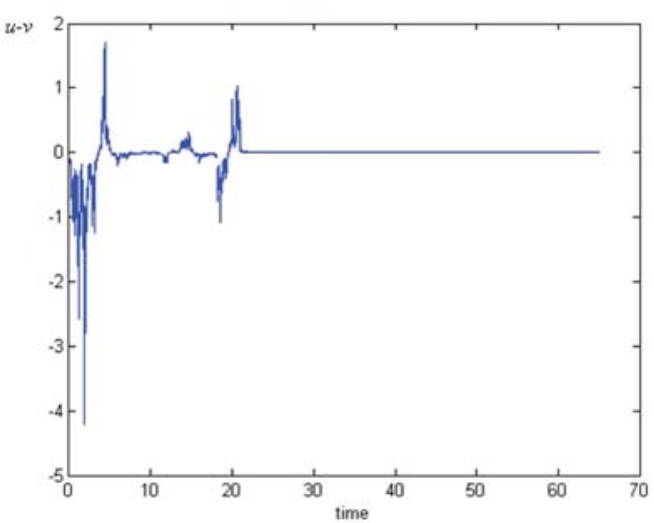

Fig. 5 Error state of the transmitter system and receiver system for $f=5 \mathrm{kHz}$

When the frequency of the information signal is increased to $10 \mathrm{kHz}$, the error signal shown in Fig. 6 is much smaller indicating that the quality of the message signal recovery is greatly improved.

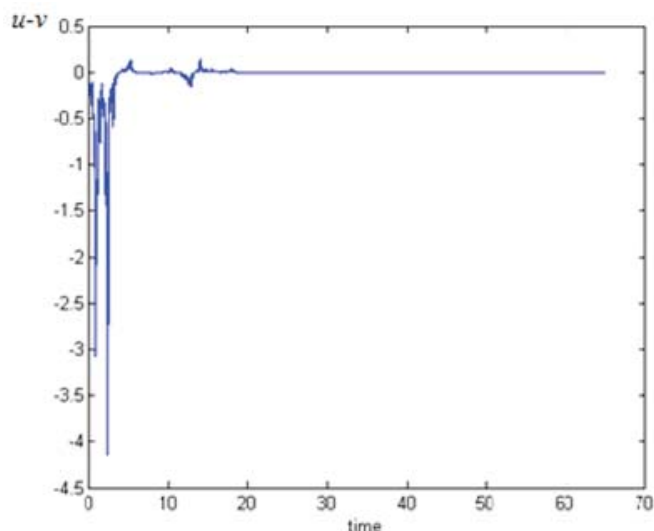

Fig. 6 Error state of the transmitter system and receiver system for $f=10 \mathrm{kHz}$

The simulation results show that, when frequency increasing, the two chaotic systems come faster in synchronisation and the oscillation signal (u-v) is smaller. This is confirmed by the graph of Fig. 7, which shows the error signal for $f=15 \mathrm{kHz}$. 


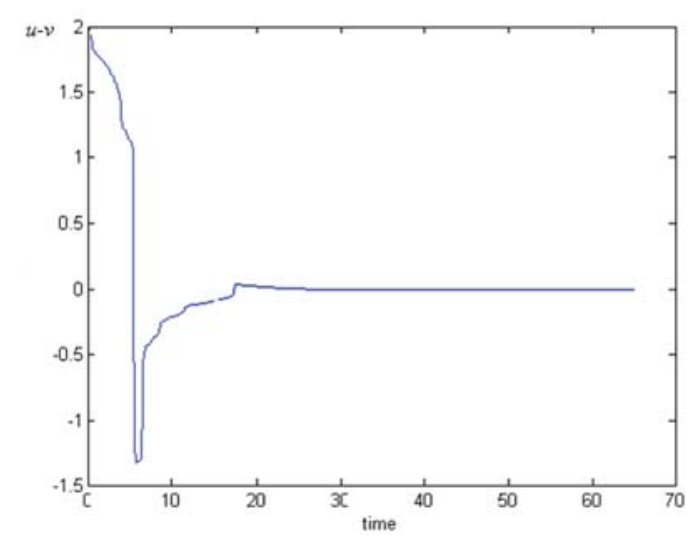

Fig. 7 Error state of the transmitter system and receiver system for $f=15 \mathrm{kHz}$

\section{Conclusions}

In this paper, chaotic masking communication system of the Duffing oscillator is designed and simulated. The proposed simula- tion model of Simulink / Matlab allows to monitor the difference between chaotic signals in the transmitter and the receiver and the synchronization time of the two systems.

According to numerical simulations, by a choice of parameters the synchronization error states converge to zero and hence the synchronization between chaotic systems is achieved.

In this paper was investigated the dependence for error signal of the frequency, that with increasing frequency the chaotic systems enter more easily into synchronisation.

Simulation results are used to visualize and illustrate the effectiveness of Duffing chaotic system in signal masking.

The results of simulatition show that the Duffing chaotic system can be used for transmission of confidental information.

\section{References}

[1] FUJISAH, H., YARNADA, T.: Stability Theory of \&Synchronized Motion in Coupied Oscillator Systems. Prog. Theor. Phys. 69, 32-47, 1953.

[2] CELIKOVSKY, S., CHEN, G.: Secure Synchronization of a Class of Chaotic Systems from a Nonlinear Observer Approach. IEEE Transactions on Automatic Control 50, 1, 2005.

[3] KOCAREV, L., PARLITZ, U.: General Afor Chaotic Synchronization with Applications to Communication. Physical Review Letters 74, 1995.

[4] JORDON, D.W., SMITH, P.: Nonlinear Ordinary Differential Equation. N. Y.: Oxford University Press, 1987.

[5] PECORA, L. M., CARROLL, T. L.: Synchronization in Chaotic Systems. Phys. Rev. Lett. 64, 8, 1990.

[6] CUOMO, K. M., OPPENHEIM, A. V.: Circuit Implementation of Synchronized Chaos with Applications to Communications. Phys. Rev. Lett 71, pp. 65-68, 1993.

[7] TENNY, R., TSIMRING, L. S., ABARBANEL, H. D. I., LARSON, L. E.: Security of Chaos-based Communication and Encryption. Digital Communications Using Chaos and Nonlinear Dynamics (Institutefor Nonlinear Science). Springer, 2006, pp. 191-229.

[8] PECORA, L. M., CARROLL, T. L.: Driving System with Chaotic Signals. Phys. Rev. Lett. A44. 1991.

[9] KOLUMBAN G., KENNEDY, M. P., CHUA, L. O.: The role of Synchronisation in Digital Communications Using Chaos - part II: Chaotic Modulation and Chaotic Synchronisation, IEEE Transactions on Circuits and Systems - I: Fundamental Theory and Applications, vol. 45, No. 11, 1998.

[10] BLEKHMAN, I.: Synchronization in Science and Technology, New York: ASME Press, 1988.

[11] JIMENEZ-TRIANA, A., WALLACE K., TANG, S., CHEN, G., GAUTHIER, A.: Chaos Control in Duffing System Using Impulsive Parametric Perturbations, IEEE Trans. Circuits and Systems - Part II, vol. 57, No. 4, pp. 305-309, 2010. 